\title{
Pituitary Infundibulum Hemangioblastoma Detected by Dynamic Enhancement MRI
}

\author{
Yong Cao, Peiyi Gao, Shuo Wang, Jizong Zhao
}

Can. J. Neurol. Sci. 2010; 37: 697-699

Hemangioblastomas in the pituitary stalk are extremely rare; only 13 cases have been described in the past. ${ }^{1-6}$ Detected by a dynamic contrast-enhanced magnetic resonance imaging (MRI), a case of pituitary infundibulum hemangioblastomas (HBL) was reported by us with tumor volume only $0.016 \mathrm{~cm}^{3}$.

\section{Case Report}

A 28-year-old woman was admitted to our hospital with a 27month history of galactorrhea and alopecia. No obvious tumor on the pituitary infundibulum was found by a cranial MRI (Figure 1), however, it indicated multiple masses in the vermis cerebellum, medulla oblongata, and dorsal portion of thoracic spinal cord 1-2 and syringomyelia from cervical to thoracic spinal cord. Previous medical history showed she was healthy and she was on no medications. Her menstruation was regular without a history of oligomenorrhea and no diabetes insipidus was found. On admission, the neurological examination revealed her slight dissociated sensory loss and slight weakness on the left hand. Her left extremities' strength was $\mathrm{V}^{-}$. The opthalmological examination was normal and abdominal computer tomography scans indicated a pancreatic cyst. Von Hippel-Lindau (VHL) disease was diagnosed based on the clinical criteria. Serum hormone levels including total thyroxine 3 , total thyroxine 4, thyroid stimulating hormone, free thyroxine 3 , free thyroxine 4 , estradiol, progesterone, human growth hormone, and testosterone were in the normal ranges. The concentration of prolactin in the serum was slightly elevated $(28.35 \mathrm{ng} / \mathrm{ml}$, normal range $1.39-24.20 \mathrm{ng} / \mathrm{ml})$, luteinizing hormone $(0.38 \mathrm{mIU} / \mathrm{ml}$, normal range $0.40-20.00 \mathrm{IU} / \mathrm{ml})$, follicle-stimulating hormone $(1.43 \mathrm{mIU} / \mathrm{ml}$, normal range $2.00-13.00 \mathrm{IU} / \mathrm{ml})$, cortisol (23.66ng/ml, normal range $50.00-250.00 \mathrm{ng} / \mathrm{ml}$ ) were slightly decreased.

A suboccipital craniotomy and a C1, T1-T3 laminectomy were performed, Macroscopically, lesions in the vermis cerebellum, medulla oblongata and thoracic spinal cord 1-2 were explored and removed completely. Hemangioblastomas were confirmed through pathologic study.

Symptoms of galactorrhea and alopecia of the patient still existed postoperatively. Magnetic resonance imaging (including dynamic contrast enhancement MRI with thin coronal scans through the sella) was performed at one week and three months after the surgery. On the second dynamic contrast-enhanced MRI, (Figure 2 a-d) a tiny enhanced mass with volume $4 \times 2 \times 2$ $\mathrm{mm}^{3}$ was detected on the pituitary infundibulum. Based on the clinical data and criteria, ${ }^{6}$ it was diagnosed as pituitary infundibulum hemangioblastoma. Three months after the surgery serum hormone levels including total thyroxine 3 , total thyroxine 4 , thyroid stimulating hormone, free thyroxine 3 , free thyroxine

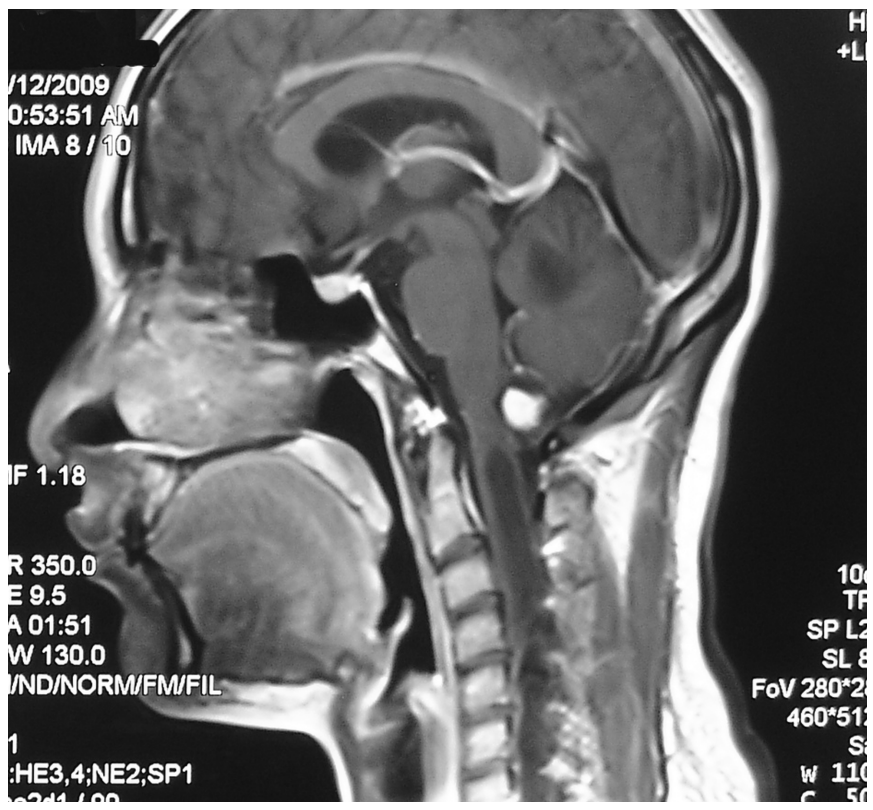

Figure 1: Preoperative sagittal T1-weighted MR images with contrast, demonstrating no obvious lesion found on the pituitary stalk.

4, luteinizing hormone, follicle-stimulating hormone, estradiol, progesterone, human growth hormone, cortisol, and testosterone were within normal ranges; the concentration of prolactin in the serum $(29.44 \mathrm{ng} / \mathrm{ml}$, normal range $1.39-24.20 \mathrm{ng} / \mathrm{ml})$ was still slightly elevated. She was prescribed $2.5 \mathrm{mg} /$ day of bromocriptine. Two weeks later the symptom of galactorrhea was relieved to some degree, and the concentration of prolactin in the serum dropped to $19.23 \mathrm{ng} / \mathrm{ml}$ (normal range 1.39 $24.20 \mathrm{ng} / \mathrm{ml})$.

\section{DISCUSSION}

Compared with pituitary stalk HBLs reported so $\operatorname{far}^{1-6}$ the tumor volume in our case was the smallest. On MRI the tumor

From the Department of Neurosurgery, Beijing Tiantan Hospital, affiliated to Capital Medical University (YC, SW, JZ); Neuroimaging center, Beijing Neurosurgical Institute (PG), Beijing, China.

Received January 25, 2010. Final Revisions Submitted March 21, 2010 Correspondence to: Jizong Zhao, Department of Neurosurgery, Beijing Tiantan Hospital, affiliated to Capital Medical University, 6 Tiantan Xili, Beijing, 100050 , China. 


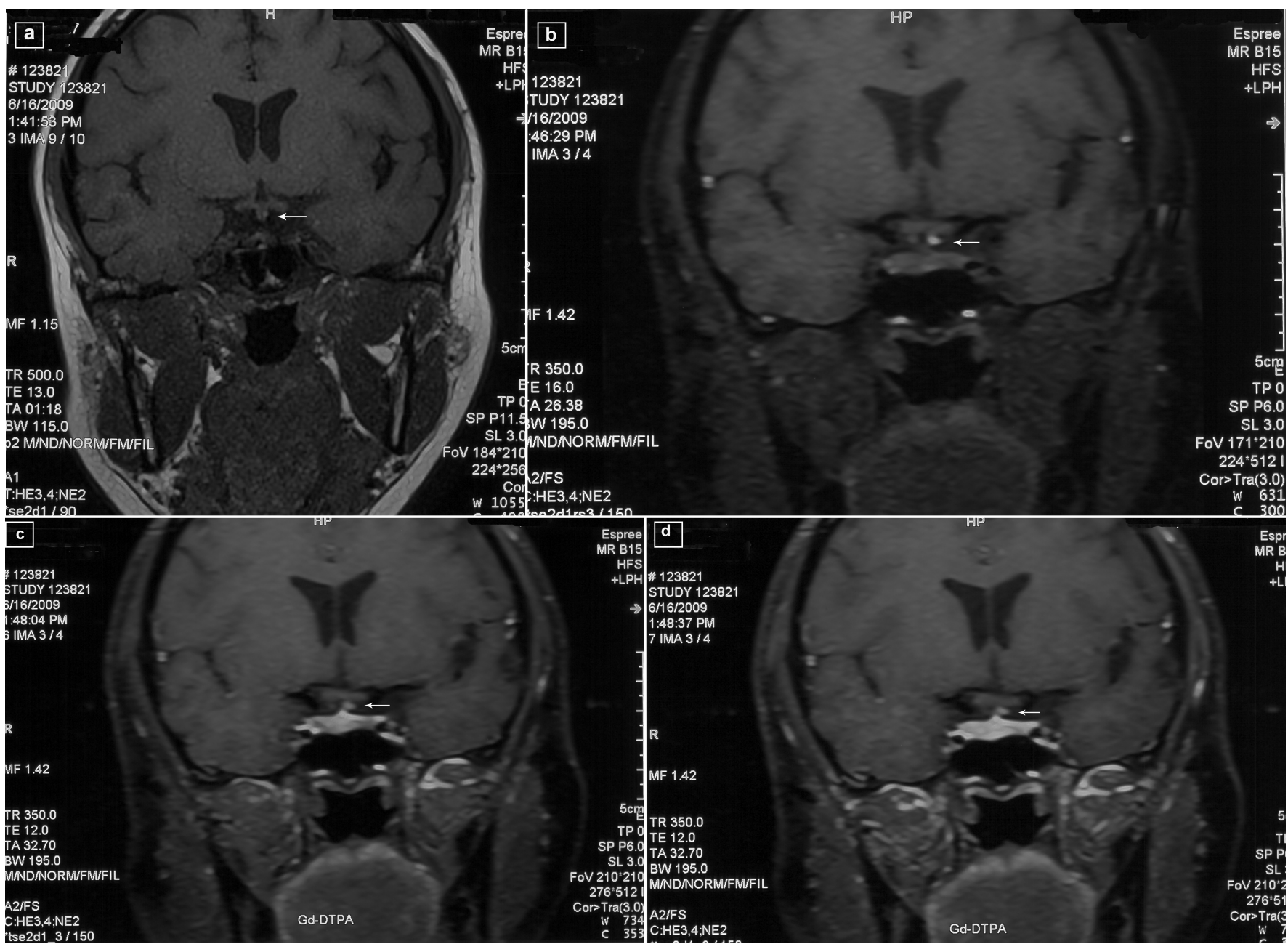

Figure 2: a) Dynamic MRI with thin coronal scans through the sella before contrast enhancement at 1:41:53 PM, showing a tiny mass (white arrow) on the left side of the pituitary infundibulum. b) Dynamic coronal contrast enhancement MRI at 1:46:29 PM, showing a tiny enhanced mass (white arrow) on the left side of the pituitary infundibulum, meanwhile the pituitary infundibulum and pituitary gland were without the enhancement. c) Dynamic coronal contrast enhancement MRI at 1:48:04 PM, showing a tiny enhanced mass (white arrow) on the left side of the pituitary infundibulum with the enhancement of the pituitary infundibulum and pituitary gland. d) Dynamic coronal contrast enhancement MRI at 1:48:37 PM, showing a tiny enhanced mass (white arrow) on the left side of the pituitary infundibulum with the enhancement of the pituitary infundibulum and pituitary gland.

could be found just to the left of the pituitary infundibulum. We could easily clarify its origin, where the tumor arose from in the pituitary stalk, because of its tiny volume.

In the Lonser's series ${ }^{6}$ these tumor volume ranged from 0.08 to $2.8 \mathrm{~cm}^{3}$, however, no patients presented with or developed signs or symptoms attributable to a pituitary stalk tumor and laboratory endocrine profiles of all patients were normal. In our case HBL volume was only $0.016 \mathrm{~mm}^{3}$ and the patient complained of the galactorrhea and alopecia that was thought as a presentation of hyperprolactinemia and hypopituitarism. Some women have excess prolactin secretion but with normal pituitary MRI. ${ }^{7}$ Our patient was in a different situation: her symptoms of galactorrhea, increase of prolactin secretion, and pituitary stalk lesion showen by the pituitary MRI corroborated each other. Therefore we speculated that the small tumor might be the etiology of the hyperprolactinemia. From a functional point of view, the tumor, even when it was very small, had influenced stalk input of hypothalamic dopamine.

In our case the tumor was too small to catch by a conventional enhancement MRI. It was the dynamic contrast enhancement MRI which helped capture the image of the tumor. Dynamic contrast enhancement MRI is performed after the administration of intravenous contrast medium to noninvasively access tumor vascular characteristics. ${ }^{8}$ It can provide useful hemodynamic information about intra-axial brain tumors that is not provided by standard MRI and therefore contributes to the differential diagnosis. ${ }^{9}$ The normal pituitary stalk lacks a blood-brain barrier, and it enhances intensely with administration of gadolinium. ${ }^{10}$ However, being a vascular tumor, hemangioblastoma has an immediately and more quickly increasing enhancement after injection of gadolinium; the hemangioblastoma's enhancement happens earlier than the pituitary stalk's when using dynamic 
contrast MRI. Therefore, dynamic contrast enhancement MRI has the capability to distinguish a tiny hemangioblastoma from the pituitary stalk.

Excluding the case presented in Neumann's report ${ }^{3}$ in which details were not described, previous cases of pituitary stalk hemangioblastomas took surgical resection or observation without surgery. ${ }^{1-6}$ In our case we chose medical treatment. Oral bromocriptine brought relief of symptoms to the patient.

Pituitary infundibulum hemangioblastoma can affect the function of the pituitary gland, even when tumor volume is very small. For hemangioblastomas, particularly in VHL disease, dynamic contrast enhancement MRI with thin coronal scans through the sella is necessary when patients present pituitary dysfunction symptoms. Oral bromocriptine can control the symptoms of hyperprolactinemia caused by pituitary infundibulum hemangioblastoma.

\section{ACKNOWLEDGMENTS}

The authors thank Dr. Bing Fu for his assistance in preparing this manuscript.

\section{REFERENCES}

1. Fomekong E, Hernalsteen D, Godfraind C, D'Haens J, Raftopoulos C. Pituitary stalk hemangioblastoma: the fourth case report and review of the literature. Clin Neurol Neurosurg. 2007; 109(3): 292-8.

2. Grisoli F, Gambarelli D, Raybaud C, Guibout M, Leclercq T. Suprasellar hemangioblastoma. Surg Neurol. 1984; 22(3): $257-62$.

3. Neumann HP, Eggert HR, Weigel K, Friedburg H, Wiestler OD, Schollmeyer P. Hemangioblastomas of the central nervous system. A 10-year study with special reference to von HippelLindau syndrome. J Neurosurg. 1989; 70(1): 24-30.

4. Kouri JG, Chen MY, Watson JC, Oldfield EH. Resection of suprasellar tumors by using a modified transsphenoidal approach. Report of four cases. J Neurosurg. 2000; 92(6): 1028-35.

5. Goto T, Nishi T, Kunitoku N, Yamamoto K, Kitamura I, Takeshima $\mathrm{H}$, et al. Suprasellar hemangioblastoma in a patient with von Hippel-Lindau disease confirmed by germline mutation study: case report and review of the literature. Surg Neurol. 2001; 56(1): 22-6.

6. Lonser RR, Butman JA, Kiringoda R, Song D, Oldfield EH. Pituitary stalk hemangioblastomas in von Hippel-Lindau disease. J Neurosurg. 2009; 110(2): 350-3.

7. Rupp D, Molitch M. Pituitary stalk lesions. Curr Opin Endocrinol Diabetes Obes. 2008; 15(4): 339-45.

8. Padhani AR. Dynamic contrast-enhanced MRI in clinical oncology: current status and future directions. J Magn Reson Imaging. 2002; 16(4): 407-22.

9. Maeda M, Itoh S, Kimura H, Iwasaki T, Hayashi N, Yamamoto K, et al. Tumor Vascularity in the Brain: Evaluation with Dynamic Susceptibility-Contrast MR Imaging. Radiology. 1993; 189(1): 233-8.

10. Simmons GE, Suchnicki JE, Rak KM, Damiano TR. MR imaging of the pituitary stalk: size, shape, and enhancement pattern. Am J Roentgenol. 1992; 159(2): 375-7. 